\title{
A SURGICAL ERROR RESULTING IN PROXIMALJUNCTIONAL KYPHOSIS IN TREATMENT OF ADOLESCENT IDIOPATHIC SCOLIOSIS
}

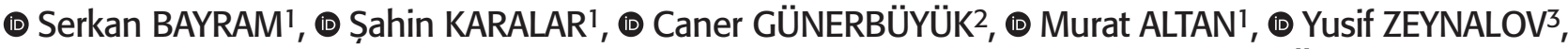 \\ ๑1 Turgut AKGÜL' ${ }^{1}$, ( Ufuk TALU'
}

\author{
${ }^{1}$ Istanbul University, Istanbul Faculty of Medicine, Department of Orthopaedics and Traumatology, Istanbul, Turkey \\ ${ }^{2}$ Koç University Faculty of Medicine, Department of Orthopaedics and Traumatology, Istanbul, Turkey \\ ${ }^{3}$ State Security Service of the Republic of Azerbaijan Medical Centre, Department of Orthopaedics and Traumatology, Baku, Azerbaijan
}

\begin{abstract}
Objective: The study aimed to investigate the incidence of painful proximal junctional kyphosis (PJK) after posterior fusion surgery in patients with adolescent idiopathic scoliosis (AIS) and their clinical results.

Materials and Methods: A total of 220 patients diagnosed with AIS (180 females and 40 males) were reviewed retrospectively. PJK was defined as the development of kyphosis more than 10 degrees between the upper instrumented end vertebra and the proximal adjacent vertebra. Visual analogue score (VAS) and the Scoliosis Research Society (SRS)-24 questionnaire were used for clinical evaluations. Thoracic kyphosis (TK) and lumbar lordosis (LL) were measured on the sagittal spinal radiograph pre and postoperatively. Computed tomography (CT) and magnetic resonance imaging (MRI) were performed on patients with pain and worst disability scores.

Results: The mean age was $15 \pm 2.4$ years and the mean follow-up period was $24.27 \pm 11.69$ months. PJK was detected in 20 of the 220 patients. TK changed from $35.5^{\circ} \pm 13.6^{\circ}$ to $25^{\circ} \pm 7.3^{\circ}$ postoperatively $(p=0.001)$ while on observation, $L L$ decreased from $53^{\circ} \pm 10^{\circ}$ to $44.4^{\circ} \pm 7.8^{\circ}$ postoperatively $(\mathrm{p}=0.001)$. The average score of the VAS average score was $3.2(3-8)$, the mean SRS-24 pain was 2.5 and the self-image score was 4.1 in patients with PJK. In three of the 20 patients with PJK, the pain was severe (VAS=8), SRS-24 pain was on average 5 and the self-image score was three in patients who had disc penetration. CT and MRI evaluations in these three patients manifested severe disc degeneration and disc space collapse caused by pedicle screw penetration through the endplate and disc.

Conclusion: Upper disc penetration with pedicle screw at the upper instrumented end vertebra leads to symptomatic disc degeneration and development of PJK. The proper placement and perfect trajectory of the most proximal pedicle screw is crucial and mandatory.

Keywords: Proximal junctional kyphosis, posterior fusion, pedicle screw, adolescent idiopathic scoliosis
\end{abstract}

\section{INTRODUCTION}

Proximal junctional kyphosis (PJK) is defined as the development of kyphosis proximal to the instrumentation in the sagittal plane. It is regularly encountered after surgeries treating kyphosis, and there are reports in the literature about its occurrence after adolescent idiopathic scoliosis surgery ${ }^{(1-7)}$. PJK is an adjacent segment problem in the upper proximal region of the instrumentation level, and the incidence rate of PJK has been reported to be between $26 \%$ and $46 \%$ in the literature ${ }^{(1-7)}$. Howbeit, there is a controversy regarding the definition of PJK, the most commonly accepted definition is a sagittal angulation of more than 10 degrees between the upper instrumented level and the proximal adjacent vertebra.
The risk factors in PJK development include, increased kyphosis before the operation, thoracoplasty, autogenous grafting for early fusion, use of pedicle screws and the level of the distal fusion being lower than L2. Studies in the literature on PJK secondary to adolescent idiopathic scoliosis (AIS) emphasise that PJK development does not have a negative effect on the quality of life, and by itself does not indicate a revision. Kim et al. ${ }^{(2)}$ have explained this with having mild adjacent segment degeneration in young patients. There is no adequate data on the indications for revision in patients with secondary PJK. The aim of the study was to investigate the incidence of painful PJK after posterior fusion surgery patients with AIS and clinical results of these patients.

Address for Correspondence: : Serkan Bayram, Istanbul University, Istanbul Faculty of Medicine, Department of Orthopaedics and Traumatology, Istanbul, Turkey E-mail: dr.serkanbayram89@gmail.com Received: 30.10.2020 Accepted: 03.11.2020

ORCID ID: orcid.org/0000-0001-7651-1200 


\section{MATERIALAND METHODS}

This study protocol was approved by the institutional review board of our institution. A retrospective study of patients with AIS who operated at a single institution was conducted. Inclusion criteria included; patients diagnosed with AIS, patients treated with posterior instrumentation and fusion using pedicle screw, had no previous spine surgery, full sets of preoperative and follow-up standing full-length anteroposterior, and lateral spinal radiographs including femoral heads and available medical records. Whereas patients who had previous spinal surgery, inadequate time for follow-up (minimum 12 months), had additional congenital deformities, diagnosed with neuromuscular disease, patients who underwent anterior spinal surgery, or osteotomy were excluded.

A total of 220 patients (180 females and 40 males) were included in the study. In all patients, the posterior ligamentous complex, facet capsule and soft tissue were preserved at the proximal end of the instrumentation. The proximal end pedicle screw was locked after correction without applying any correction force.

Concerning the sagittal plane analysis, a sagittal spinal radiograph was used to measure the thoracic kyphosis (TK), lumbar lordosis (LL) and central sagittal vertical line (CSVL). The development of kyphosis, more than 10 degrees, between the upper proximal instrumented level and the adjacent proximal vertebra was defined as PJK. Visual analogue score (VAS) and SRS-24 questionnaires were used to determine the correlation of clinical complaints. Computed tomography (CT) and magnetic resonance imaging (MRI) were performed on patients with pain and worst disability scores.

\section{Statistical Analysis}

All statistical analyses in this study were performed using SPSS version 24.0 (IBM Corp., Armonk, NY). Study data were
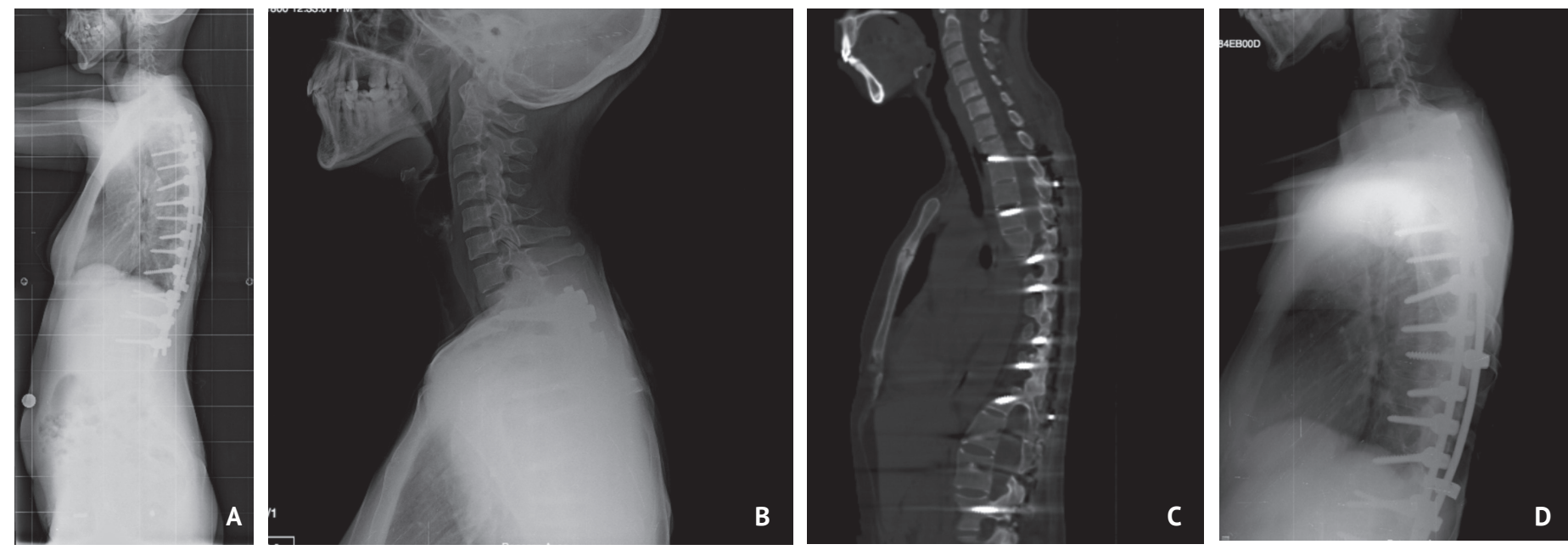

Figure 1. Patient underwent posterior fusion surgery that has pain at the cervicothoracic junction. The sagittal spinal radiograph showed proximal disc degeneration and kyphosis (A, B). CT examination showed disc space collapsed and pedicle screw penetration (C). Instrumentation was revised and lengthened one level above (D)

$\mathrm{CT}$ : Computed tomography 


\section{DISCUSSION}

For the correction of spinal deformities, instrumentation through a posterior approach and fusion yielded successful outcomes. Nevertheless, the development of PJK in the vertebrae adjacent to the instrumented vertebra in rigid systems has emerged as a severe problem. In the literature, there is no established consensus on the diagnosis and incidence of PJK. Lee et al. ${ }^{(7)}$, in their study, have reported an incidence of $46 \%$ while the latest studies report it to be $26 \%(2,5,7,8)$. PJK after surgery for AIS has been defined by Lee et al..$^{(7)}$, to be the development of kyphosis more than 5 degrees proximal to the implant. Whereas, Glattes et al.(6) defined it as the angle between the first instrumented vertebra endplate and the adjacent proximal vertebra upper endplate being more than 10 degrees. Helgeson et al.(5) recommend that the threshold should be 15 degrees, and the measurement should be performed between the endplates of two proximal vertebrae. The reported rates of PJK differ depending on the definition. Considering the published spectrum of PJK definition in AIS, in our series of patients, the incidence of PJK after surgery was determined to be $13 \%$.

PJK development after adult spine deformities was investigated in more detail, and risk factors were determined. These factors included patients older than 55 years of age with combined anterior and posterior surgery, independent of surgical errors ${ }^{(9)}$, increased kyphosis before the operation, thoracoplasty, autogenous grafting for early fusion, use of pedicle screws and the level of the distal fusion being lower than $L 2^{(1-7)}$. In our series, patients who needed revision surgery did not have any of the risk factors reported in the literature, but PJK was developed based on disc degeneration and loss of disc height. Disc degeneration and penetration of the pedicle screws into the disc were demonstrated with MRI scans. Although the literature suggests that revision is not needed in patients with secondary PJK; however, revision surgery is necessary for pain relief when PJK is accompanied by disc degeneration and collapse. Surgical inclusion of the degenerated and painful proximal disc and segment into the fusion area resulted in significant relief of the patients' complaints.

Meter et al. ${ }^{(10)}$ have shown in their cadaveric studies that in order for the pedicle screws inserted, especially at the lumbar area, not to cause endplate damage, the tip must lie at least $3 \mathrm{~mm}$ from the endplate. In their anatomic study, Melrose et al.(11) have demonstrated disc degeneration with mechanic destabilisation through injuring annulus fibrosis. Notwithstanding, although Meter et al. ${ }^{(10)}$ have advised that pedicle screws cause centripetal
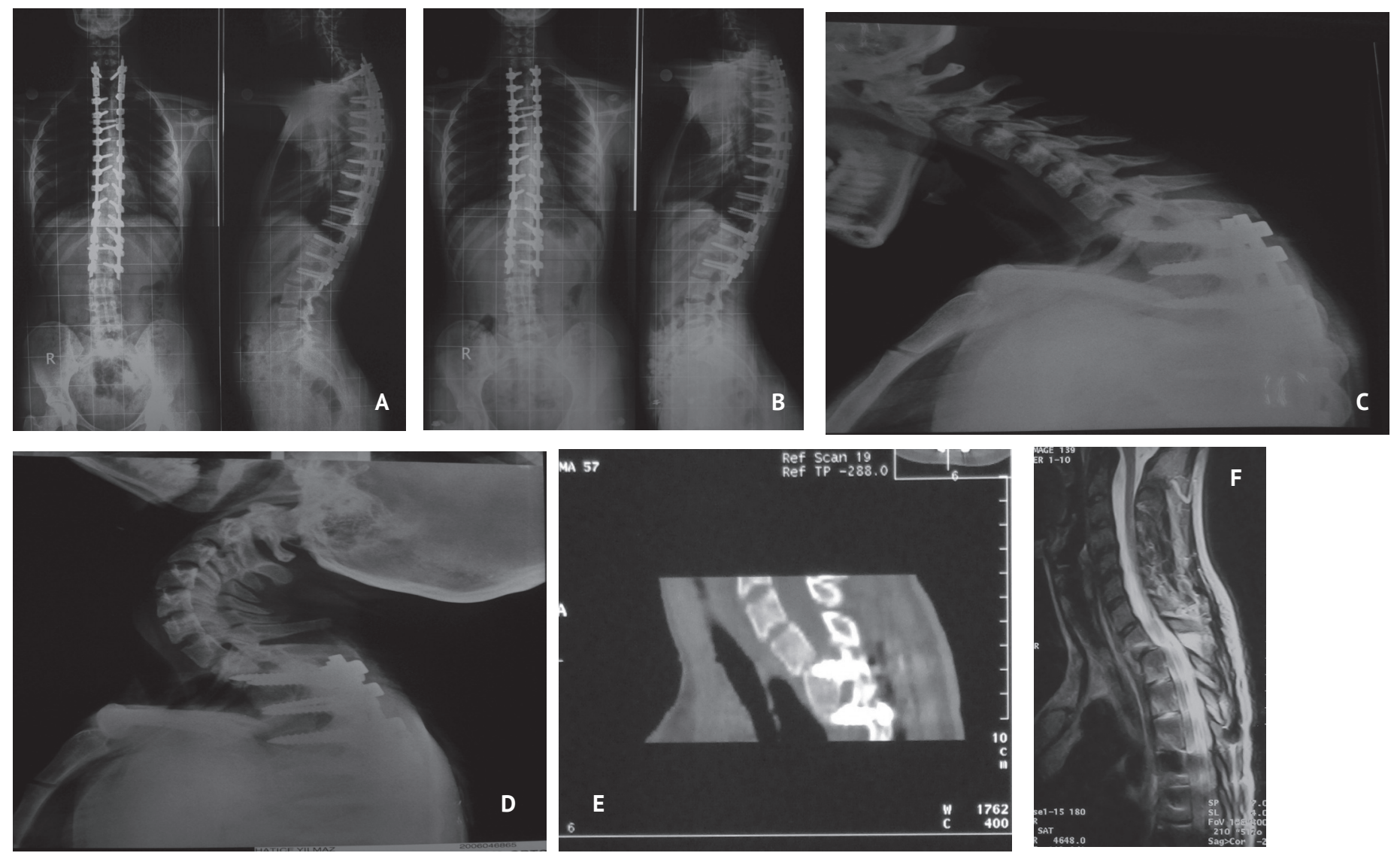

Figure 2. Another patient with PJK and cervical pain diagnosed at follow-up visit (A). Lateral flexion and extension radiographs of cervical spine showed no major instability at the PJK side (B,C). CT and MRI showed disc space collapsed and screw penetration at most proximal side (D, E). Patient who underwent revision surgery (F)

PJK: Proximal junctional kyphosis, CT: Computed tomography, MRI: Magnetic resonance imaging 
turkishspine

damage and in turn prevents the release of phospholipase located in the nuclear substance, and the presence of a metallic screw inside the disc causes damage to it, the consequences of these in the mobile discs could not be clearly understood. In our study, pedicle screws penetrating the endplate and the disc consistently resulted in symptomatic degeneration and loss of height. Even if centripetal, the presence of metallic materials in the disc causes disc degeneration characterised by pain.

\section{Study Limitations}

There were some limitations in the study. Firstly, it is a retrospective study with a small number of patients. Secondly, other sagittal spinal measurements, such as pelvic tilt, sacral slope and cervical lordosis, were not investigated pre and postoperatively. Thirdly, the follow-up period was shorter. Patients may develop the PJK for a long time after the surgery. And finally, we did not classify our patients according to the Lenke classification system; thus, our cohort was not homogeneous.

\section{CONCLUSION}

We emphasised that, utmost care should be taken while using the pedicle screws to ensure a perfect placement and trajectory of it at the uppermost vertebra to avoid penetration of proximal adjacent endplate and disc, to prevent the development of painful PJK and the need for revision surgery. The use of proper imaging is crucial during the insertion of these specific pedicle screws.

\section{Ethics}

Ethics Committee Approval: Since the nature of our study was retrospective, study was conducted with institutional review board approval, not ethics committee.

Informed Consent: Retrospective study.

Peer-review: Internally peer-reviewed.

\section{Authorship Contributions}

Concept: T.A., Design: S.B, Data Collection or Processing: Ş.K., M.A., Analysis or Interpretation: C.G, Literature Search: Y.Z, T.A, U.T, Writing: T.A., S.B.
Conflict of Interest: The authors declare that they have no conflict of interest.

Financial Disclosure: There is no funding source.

\section{REFERENCES}

1. Kim YJ, Bridwell KH, Lenke LG, Kim J, Cho SK. Proximal junctional kyphosis in adolescent idiopathic scoliosis following segmental posterior spinal instrumentation and fusion: Minimum 5-year followup. Spine (Phila Pa 1976). 2005;30:2045-50.

2. Kim YJ, Lenke LG, Bridwell KH, Bridwell KH, Kim, Cho SK, et al. Proximal junctional kyphosis in adolescent idiopathic scoliosis after 3 different types ofposterior segmental spinal instrumentation and fusions: Incidence and risk factor analysis of 410 cases. Spine (Phila Pa 1976). 2007; 32:2731-8.

3. Hollenbeck SM, Glattes RC, Asher MA, Lai SM, Burton DC. The prevalence of increased proximal junctional flexion following posterior instrumentation and arthrodesis for adolescent idiopathic scoliosis. Spine (Phila Pa 1976). 2008;33:1675-81.

4. Wang J, Zhao Y, Shen B, Wang C, Li M. Risk factor analysis of proximal junctional kyphosis after posterior fusion in patients with idiopathic scoliosis. Injury. 2010;41:415-20.

5. Helgeson MD, Shah SA, Newton PO, Clements DH, Betz RR, Marks MC, et al. Harms Study Group: Evaluation of proximal junctional kyphosis in adolescent idiopathic scoliosis following pedicle screw, hook, or hybrid instrumentation. Spine (Phila Pa 1976). 2010;35:177-81.

6. Glattes RC, Bridwell KH, Lenke LG, Kim YJ, Rinella A, Edwards C II. Proximal junctional kyphosis in adult spinal deformity following long instrumented posterior spinal fusion: Incidence,outcomes, and risk factor analysis. Spine (Phila Pa 1976). 2005;30:1643-9.

7. Lee GA, Betz RR, Clements DH III, Huss GK. Proximal kyphosis after posterior spinal fusion in patients with idiopathic scoliosis. Spine (Phila Pa 1976). 1999;24:795-9.

8. Cho SK, Kim YJ, Lenke LG. Proximal Junctional Kyphosis Following Spinal Deformity Surgery in the Pediatric Patient. J Am Acad Orthop Surg. 2015;23:408-14.

9. Kim Y], Bridwell KH, Lenke LG, Glattes CR, Rhim S, Cheh G. Proximal junctional kyphosis in adult spinal deformity after segmental posterior spinal instrumentation and fusion: minimum five-year follow-up. Spine (Phila Pa 1976). 2008;33:2179-84.

10. Meter JJ, Polly DW, Miller DW, Popovic NA, Ondra SL. A method for radiographic evaluation of pedicle screw violation of the vertebral Endplate Spine (Phila Pa 1976). 1996;13:1587-92.

11. Melrose J, Shu C, Young C, Ho R, Smith MM, Young AA et al. Mechanical destabilization induced by controlled annular incision of the intervertebral disc dysregulates metalloproteinase expression and induces disc degeneration. Spine (Phila Pa 1976). 2012;37:18-25. 